\title{
Les alluvions anciennes de la Meurthe en Lorraine sédimentaire (Est du bassin de Paris, France) : étude morphosédimentologique et essai de reconstitution paléoclimatique
}

Pleistocene alluvial deposits of the Meurthe river in Lorraine (Eastern Paris basin, France): morpho-sedimentology and palaeoclimatic reconstruction Die ältesten Schotter der Meurthe in Lothringen (Osten des Pariser Beckens, Frankreich) : morphosedimentologische Untersuchung und Versuch einer Rekonstruktion der Paläoklimate

Stéphane Cordier, Dominique Harmand et Monique Beiner

\section{(2) OpenEdition}

Journals

Édition électronique

URL : http://journals.openedition.org/rge/2736

DOI : $10.4000 /$ rge. 2736

ISSN : 2108-6478

Éditeur

Association des géographes de l'Est

Édition imprimée

Date de publication : 1 septembre 2002

ISSN : 0035-3213

Référence électronique

Stéphane Cordier, Dominique Harmand et Monique Beiner, « Les alluvions anciennes de la Meurthe en Lorraine sédimentaire (Est du bassin de Paris, France) : étude morphosédimentologique et essai de reconstitution paléoclimatique », Revue Géographique de l'Est [En ligne], vol. 42 / 4 | 2002, mis en ligne le 17 janvier 2011, consulté le 08 septembre 2020. URL : http://journals.openedition.org/rge/2736 ; DOI : https://doi.org/10.4000/rge.2736

Ce document a été généré automatiquement le 8 septembre 2020

Tous droits réservés 


\title{
Les alluvions anciennes de la
} Meurthe en Lorraine sédimentaire (Est du bassin de Paris, France) : étude morphosédimentologique et essai de reconstitution paléoclimatique

\author{
Pleistocene alluvial deposits of the Meurthe river in Lorraine (Eastern Paris \\ basin, France): morpho-sedimentology and palaeoclimatic reconstruction \\ Die ältesten Schotter der Meurthe in Lothringen (Osten des Pariser Beckens, \\ Frankreich) : morphosedimentologische Untersuchung und Versuch einer \\ Rekonstruktion der Paläoklimate
}

Stéphane Cordier, Dominique Harmand et Monique Beiner

Depuis plusieurs décennies, de nombreux travaux se sont attachés à dégager les grandes caractéristiques de l'alluvionnement en Lorraine sédimentaire. La plupart des recherches a ainsi porté sur les vallées de la Meuse et de la Haute Moselle à l'amont de Toul (Vaskou, 1981; Taous, 1994) et sur le site de capture de la Moselle près de cette dernière localité (Harmand et Le Roux, 2000). Malgré un certain nombre d'études, de nombreuses lacunes subsistent en revanche pour ce qui concerne la connaissance des autres vallées lorraines, notamment de celle de la Meurthe (Théobald et Gardet, 1935 ; Tricart, 1948 ; Bonnefont, 1986 ; Kowalski 1988 ; Kowalski et al., 1990 ; Weisrock 1990 et 1997 ; Buzzi et al., 1993 ; Vogt, 1992), qui se prolongeait jusqu'au Rhin suivant l'actuelle vallée mosellane avant la capture de la Moselle (Kremer, 1954 ; Schirmer, 1990), et constituait ainsi un axe de drainage essentiel en Lorraine, la Paléo-Meurthe (Harmand et al., 1995). Le présent travail, par le recours à diverses méthodes géomorphologiques et sédimentologiques (granulométrie, pétrographie, étude de minéraux lourds inédite 
dans le bassin de la Meurthe) a pour but de dégager les principales caractéristiques de l'alluvionnement de la Meurthe et de mieux appréhender l'organisation du dispositif alluvial.

Le secteur étudié, long de $70 \mathrm{~km}$, correspond à la vallée de la Meurthe en Lorraine sédimentaire méridionale, depuis Baccarat jusqu'à la confluence avec la Moselle à Pompey. Cette section se trouve au débouché des Vosges cristallines et gréseuses.

Entre Baccarat et Pompey (fig. 1 et 2), la vallée se développe dans des formations secondaires du Bassin parisien et recoupe les cuestas du Muschelkalk, de l'Infralias et du Bajocien (respectivement à l'aval de Baccarat, à Rosières-aux-Salines et à l'aval de Nancy). Un dernier affleurement de roches résistantes est développé dans les calcaires du Muschelkalk à l'aval de Lunéville, dans la traversée de l'anticlinal de Mont-surMeurthe (Le Roux, 1980).

Entre ces seuils correspondant à une vallée étroite (parfois moins d'un kilomètre), la vallée de la Meurthe s'élargit dans les assises marneuses du Keuper et du Lias, dépassant parfois $5 \mathrm{~km}$ de largeur. Ces secteurs de vallée large coïncident avec trois bassins alluviaux (Mondon, Vitrimont et Nancy, fig. 1 et 2) plus ou moins vastes (25 à 70 $\mathrm{km} 2)$.

Figure 1 : Cadre géologique et morphostructural du bassin versant de la Meurthe

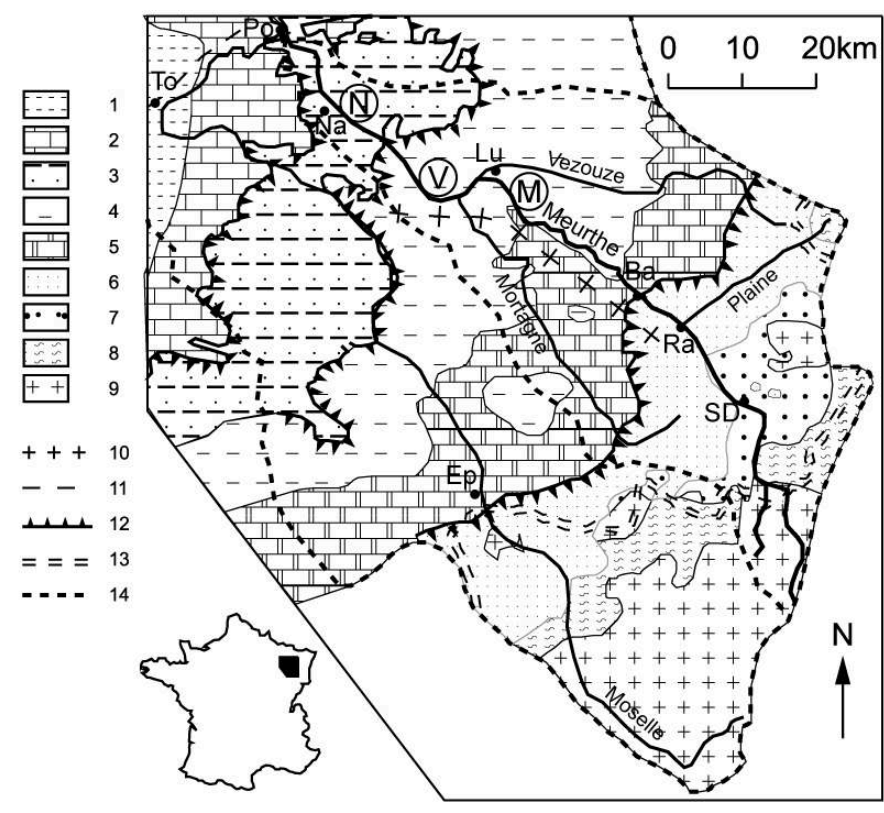

1, marnes (Bathonien-Callovien); 2, calcaires (Bajocien); 3, marnes et grès (Lias); 4, marnes et dolomies (Keuper); 5 , calcaires (Muschelkalk); 6, grès et conglomérats (Buntsandstein); 7, grès et argiles (Permien); 8 , socle métamorphique et volcano-sédimentaire; 9 , socle granitique; 10 , axe anticlinal; 11 , axe synclinal; 12, front de cuesta; 13 , limites de l'extension maximale des glaces au Weichsélien (d'après Mercier
et al., 1999); 14 , limite de bassin versant. (M), (M). (N): 14, limite de bassin versant.

Vitrimont et Nancy

Ba = Baccarat, Ep=Epinal, Lu= Lunéville, $\mathrm{Na}=$ Nancy, Po=Pompey, Ra= Raon l'Etape, SD=Saint-Dié-desVosges, To=Toul. 
Figure 2 : Les terrasses de la Meurthe entre Baccarat et Pompey

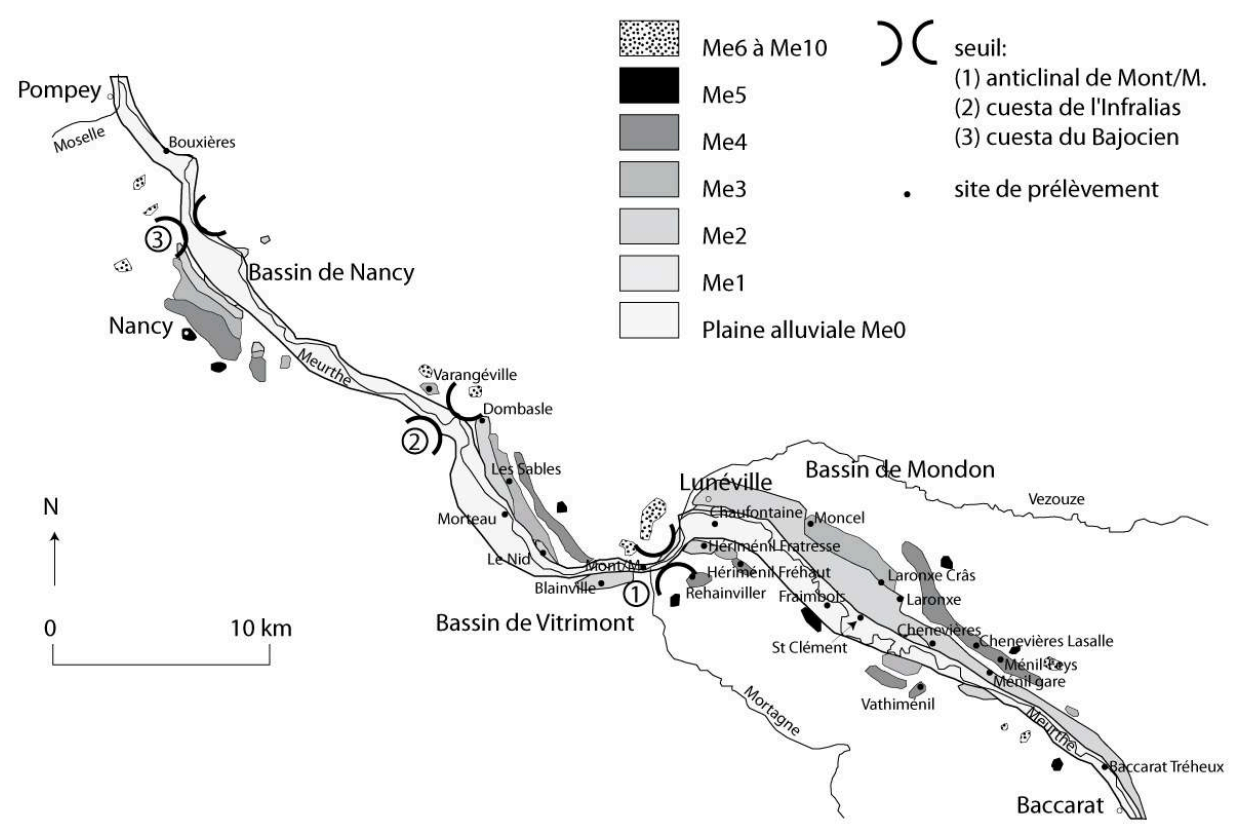

\section{Le dispositif alluvial de la Meurthe en Lorraine sédimentaire}

Des observations de terrain, l'analyse de Modèles Numériques de Terrain et l'exploitation de plusieurs centaines de sondages (Bureau des Recherches Géologiques et Minières et sources diverses) permettent de reconnaître 10 niveaux de terrasses étagés (fig. 3) au-dessus de la plaine alluviale Me0. Ces niveaux sont notés de Me1 (basse terrasse) à Me10 pour le plus ancien. Les sommets de ces niveaux sont situés à des altitudes relativement constantes, respectivement à + 3-5 m au-dessus du talweg (Me1), + $12 \mathrm{~m}$ (Me2), + $20 \mathrm{~m}$ (Me3), + 30-35 m (Me4), + $45 \mathrm{~m} \mathrm{(Me5),} \mathrm{+} 60 \mathrm{~m}$ (Me6), + $70 \mathrm{~m} \mathrm{(Me7),}$ + $85 \mathrm{~m}$ (Me8), + 110-120 m (Me9), et + $160 \mathrm{~m}$ (Me10). 
Figure 3 : Profils longitudinal (a) et transversal (b) des niveaux de terrasses de la Meurthe en Lorraine sédimentaire
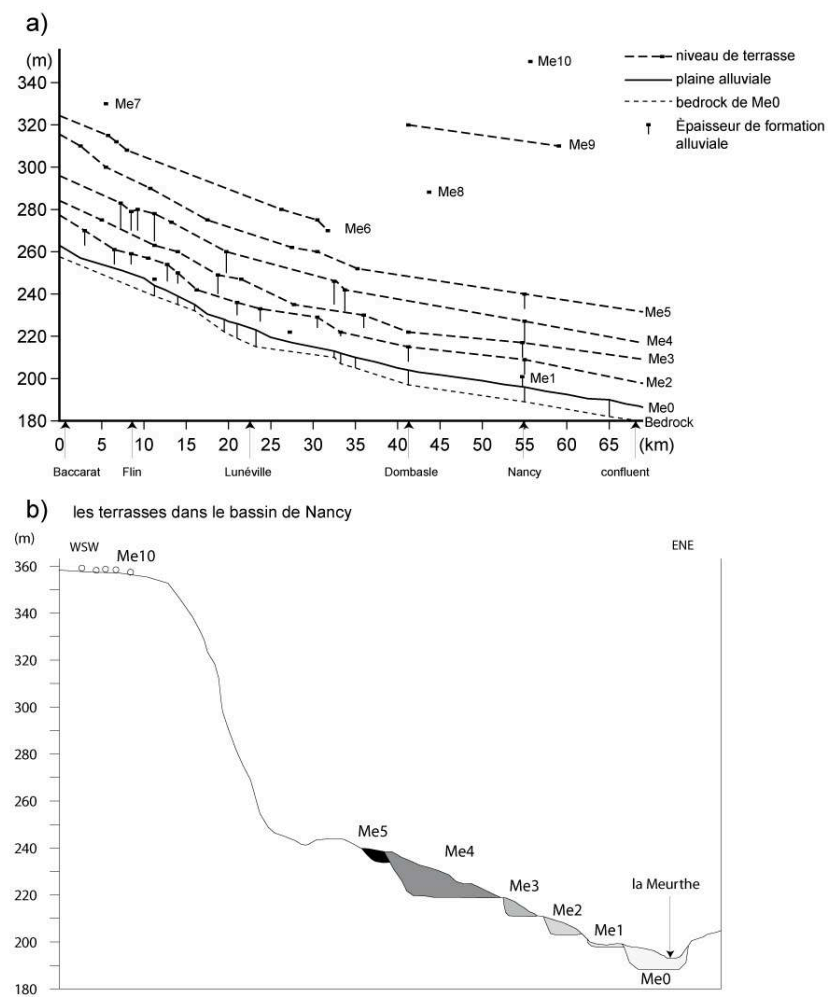

6 Les niveaux les plus récents (de Me0, la plaine alluviale, à Me5) correspondent à 6 formations alluviales (Lebret et al., 1993) notées de F0 (remblaiement de fond de vallée) à F5. Au-dessus ne subsistent que des alluvions fortement altérées ou résiduelles, les plus élevées (niveau Me10) se trouvant au sommet de la cuesta du Bajocien. L'ensemble de ces niveaux est représenté sur un profil longitudinal couvrant l'ensemble du secteur d'étude (fig. 3). Le parallélisme entre les niveaux, déjà observé dans la vallée de la Haute Moselle-Meuse (Harmand et al., 1998), traduit l'absence de déformation locale importante au Quaternaire (Le Roux et Harmand, 1998).

Les formations F2 à F5 correspondent à des dépôts de plusieurs mètres d'épaisseur. Les coupes les plus importantes se trouvent dans le bassin de Mondon développé sur l'interfluve Meurthe-Vezouze. Elles coïncident avec la formation F4, qui constitue ainsi un niveau-repère.

Plusieurs coupes ont également pu être étudiées dans les formations F2, F3 et F5. Elles complètent les données déjà recueillies sur la plaine alluviale Me0 (Havard et al., 1970 ; Carcaud, 1992) et permettent des comparaisons avec le niveau Me4. Aucune étude n'a en revanche pu être menée sur la basse terrasse Me1 (+3-5 m), d'extension réduite et qui n'est identifiée que très localement (près de Lunéville et de Nancy).

\section{Le niveau-repère Me4 dans le bassin de Mondon}

Cinq grandes coupes (fig. 4) se répartissent sur l'ensemble du bassin de Mondon et permettent l'observation des alluvions sur une épaisseur de 6 à $9 \mathrm{~m}$. Ces coupes sont situées en bordure d'un replat topographique situé à 30-35 m au-dessus du fond de vallée. 
Figure 4 : Représentation schématique des principales coupe de niveau Me4 dans le bassin de Mondon

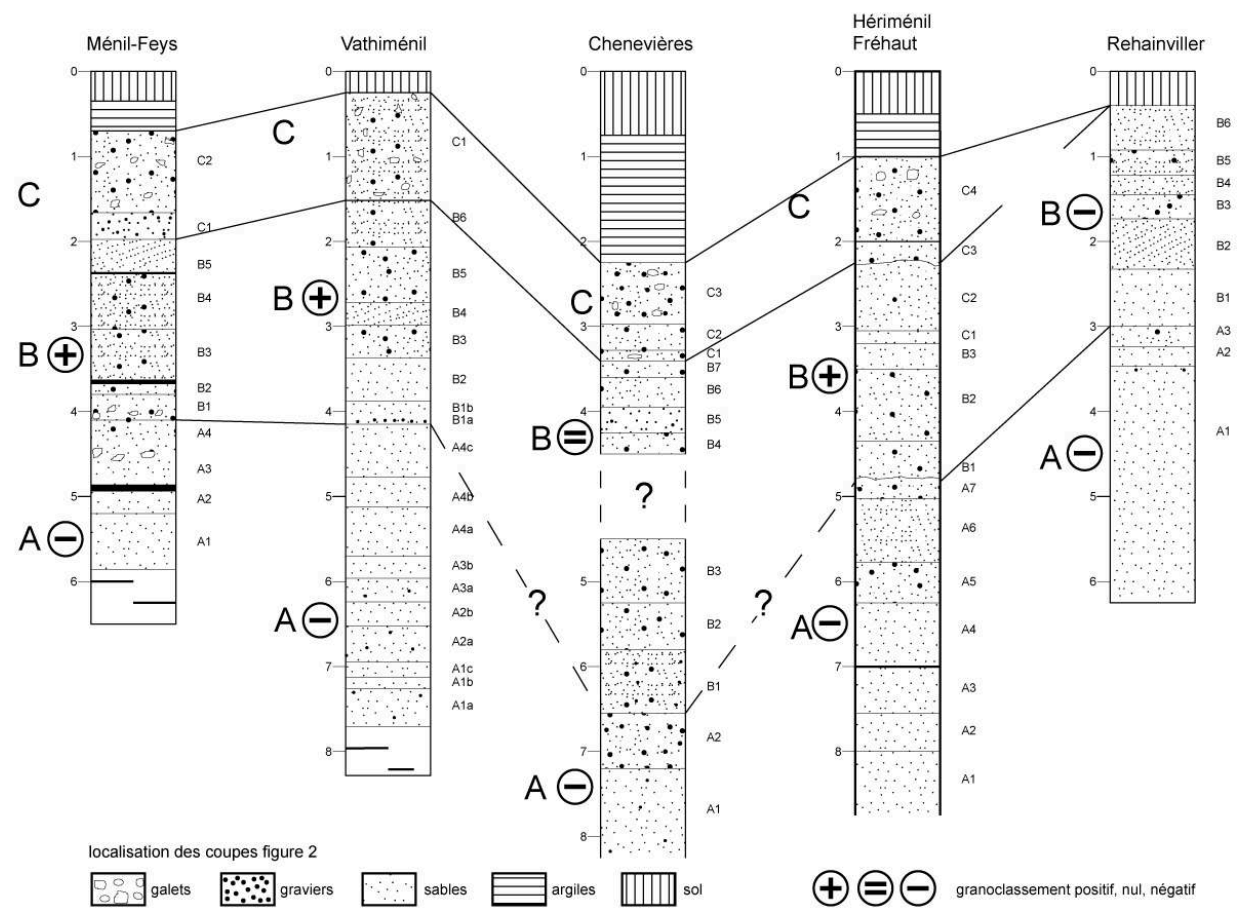

\section{A. Granularité et structures sédimentaires des alluvions de la formation F4 (Me4)}

Ces coupes présentent des faciès très proches: les alluvions sont essentiellement sableuses ou sablo-graveleuses sur la plus grande épaisseur du dépôt (fig. 4). Les galets ne sont visibles que sur une épaisseur de 1 à $2 \mathrm{~m}$ en sommet de remblaiement (hormis à Rehainviller où l'on n'observe que quelques galets dispersés dans les séquences sableuses). A Ménil-Feys, deux bancs grossiers peu épais sont par ailleurs visibles dans les séquences inférieures plus fines. Ces alluvions sont localement recouvertes par une formation limono-sableuse, peu épaisse et fortement pédogénisée.

Trois séries peuvent être distinguées : à la base, la série $\mathrm{A}$, épaisse de 2 à $4 \mathrm{~m}$, est formée de fines séquences sableuses (souvent plus de $70 \%$ de sables), et se caractérise par un granoclassement négatif (enrichissement progressif en graviers vers le haut). La série B (épaisseur 2-3 m) est plus graveleuse (plus de 20 voire $30 \%$ de graviers) et présente un granoclassement souvent positif. La granularité des éléments reste cependant modeste, les sables représentant encore plus de $60 \%$ des éléments : avec cette prédominance des éléments sableux - s'expliquant notamment par le piégeage des éléments grossiers dans le bassin de Saint-Dié (Carcaud, 1992), les séries A et B traduisent ainsi la dynamique d'une rivière à régime d'écoulement modéré.

Moins épaisse ( 1 à $2 \mathrm{~m}$ ), la série supérieure $\mathrm{C}$ est plus riche en dépôts grossiers (10 à $30 \%$ de galets, moins de $40 \%$ de sables) : sa mise en place marque un changement important dans la dynamique fluviale de la Meurthe dont la compétence augmente sensiblement - même si la taille des galets reste modeste, excédant rarement $6 \mathrm{~cm}$. 
13 Dans le bassin de Mondon, l'ensemble du dépôt présente donc un granoclassement globalement négatif.

L'étude des structures sédimentaires confirme l'opposition entre des niveaux sablograveleux et grossiers: les niveaux sableux présentent un litage subhorizontal, à l'exception de certaines séquences de la série B où s'observent des stratifications obliques entrecroisées. L'observation d'un chenal de recoupement caractéristique des rivières à tresses (Doeglas, 1962; Marc Durand, comm. orale) dans la coupe de Vathiménil, la disposition générale des alluvions en séquences peu épaisses permettent de conclure que la mise en place des dépôts s'est effectuée dans un contexte de tressage (Allen, 1982 ; Miall, 1996). Cette donnée rejoint celles obtenues pour les alluvions des moyennes terrasses de la Moselle à sa sortie du Massif vosgien (Taous, 1994), et confirme la capacité de la Meurthe à développer une dynamique de tressage, déjà mise en évidence pour le remblaiement de fond de vallée (Carcaud, 1992).

Le dispositif des séries alluviales confirme cette conclusion: l'ensemble du banc grossier $\mathrm{C}$ présente de fait une base nettement érosive, et tronque les séquences supérieures de la série B à litage subhorizontal. Aucune figure sédimentaire n'apparaît dans le dépôt grossier, à l'exception de glosses et plaquettes argileuses au contact avec les horizons limoneux sus-jacents. Ces figures, témoignant d'une évolution pédogénétique sous conditions périglaciaires (Caillier, 1977), sont néanmoins difficiles à rattacher au système alluvial (Gury, 1990 et comm. orale).

\section{B. Une origine contrastée des matériaux}

\section{Les apports de l'étude des sables}

16 L'examen à la loupe binoculaire montre que les sables des trois séries $\mathrm{A}, \mathrm{B}$ et $\mathrm{C}$ sont exclusivement siliceux. Les quartz représentent généralement plus de $80 \%$ des grains, le reste de la fraction étant pour l'essentiel composé de feldspaths, de micas ainsi que de minéraux denses. Cette composition traduit le rôle fondamental joué par le bassin vosgien de la Meurthe (amont de Baccarat) dans la fourniture en sédiments. L'examen de la forme et de l'état de surface des grains montre quant à lui des proportions significatives de non-usés et d'émoussés luisants, les ronds mats étant plus rares. Si le rapprochement entre les ronds mats (et dans une moindre mesure les émoussés luisants) et les grès, de même que celui entre les non-usés et le socle cristallin sont souvent pratiqués - à juste titre -, il convient cependant ici de rester prudent sur la signification de cette répartition, la couverture gréseuse pouvant fournir des grains non-usés (quartz de néogenèse). En l'absence d'étude approfondie, il est donc seulement possible d'établir que les sables proviennent à la fois de la couverture grésoconglomératique et du socle cristallin.

17 Les déterminations des minéraux lourds réalisées par Monique Beiner (plus de 200 grains par échantillon) permettent de mieux préciser l'origine des alluvions sableuses. Les trois principaux éléments (fig. 5) sont la tourmaline (proportion moyenne : $35 \%$ ), l'amphibole (27\%) - amphibole verte, et amphibole brune-verte à brune, typique des roches granitiques vosgiennes - et le zircon (21\%), qui apparaît souvent sous sa forme zonée, attestant de son origine siliceuse. 
Figure 5 : Spectres de minéraux lourds des alluvions de la Meurthe

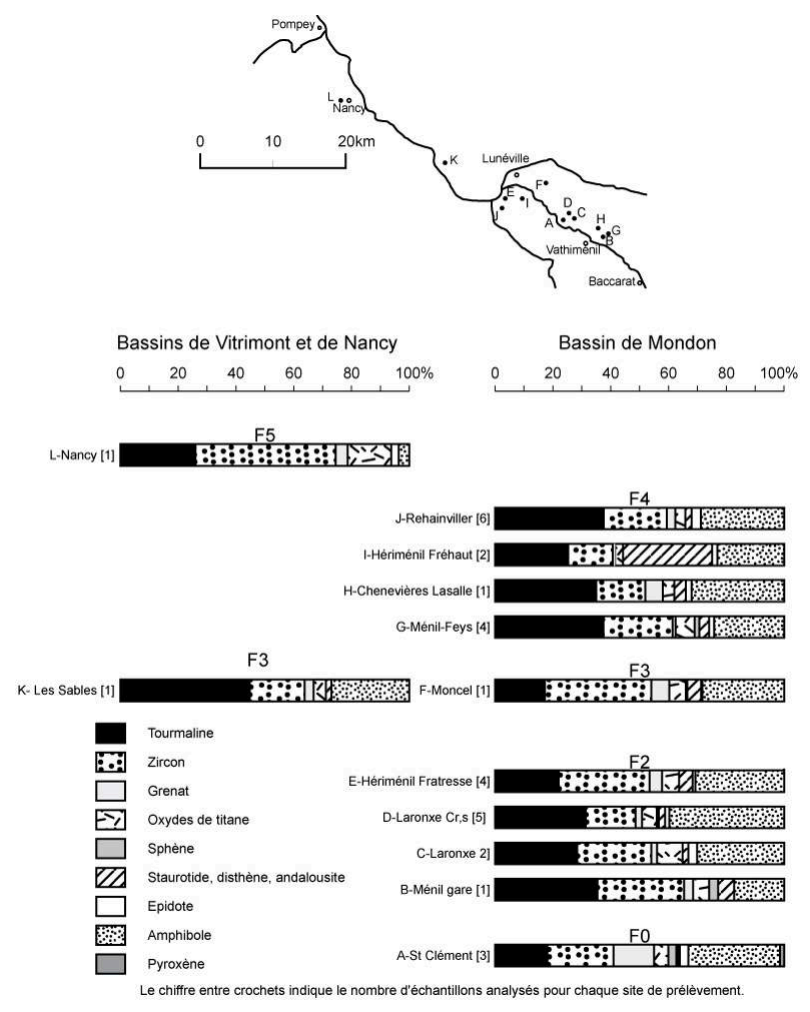

18 Alors que l'amphibole provient essentiellement du socle cristallin (Hameurt, 1967), le zircon et surtout la tourmaline sont des minéraux particulièrement abondants dans les grès et conglomérats du Permo-Trias (Perriaux, 1961). Leur prédominance dans les alluvions, de même que la faible proportion, notamment dans les niveaux récents, en grenat (minéral très présent dans le socle vosgien), montre que les apports sableux proviennent essentiellement de la couverture gréso-conglomératique.

19 Cette conclusion est en accord avec les conditions de mise en place des séries A et B précédemment évoquées (rivière à dynamique fluviale modérée). Cette importance de la couverture gréseuse peut notamment être expliquée par la lithologie : plus de $75 \%$ de l'ensemble du bassin vosgien de la Meurthe est développé dans les formations gréseuses, et la proportion avoisine encore $64 \%$ si l'on exclut les bassins, également gréseux dans leur partie amont, de la Mortagne et de la Vezouze (qui ne rejoignent la Meurthe qu'à l'aval du bassin de Mondon).

\section{L'étude des galets}

20 La réalisation de comptages lithologiques sur les galets de $20-40 \mathrm{~mm}$ de diamètre (seule dimension étudiée du fait de la rareté des galets de plus grande taille) prélevés dans les séries A et surtout $C$ permet de dégager les enseignements suivants (fig. 6) :

21 - l'origine exclusivement vosgienne des alluvions se retrouve : les galets sont issus $\mathrm{du}$ socle (granites) ou de la couverture gréseuse (quartz, quartzite, grès) ;

22 - les galets de la série $\mathrm{C}$ de la formation $\mathrm{F} 4$ correspondent pour une nette majorité (50 à $80 \%$ ) à des éléments cristallins issus du haut bassin de la Meurthe; on constate simplement une tendance à la diminution vers l'aval, liée à l'altération des granites au cours du transport. 
23 En revanche, le banc grossier intercalé dans les niveaux sableux à Ménil-Feys (série A) montre quasi-exclusivement des apports de couverture, rejoignant en cela les résultats de l'étude minéralogique. Ce contraste accentue l'idée d'une rupture dans la dynamique fluviale et dans la provenance du matériel entre les séries inférieures (A et B) et la série C.

24 Enfin, les galets de socle de la série $C$ apparaissent comme très altérés, plus de la moitié d'entre eux étant friables ou pourris (fig. 6). Au-delà de l'ancienneté relative de F4, l'explication est à rechercher dans leur position en sommet de remblaiement, à proximité des horizons affectés par la pédogenèse.

Figure 6 : Pétrographie et altération des galets de la Meurthe (20-40 mm de diamètre)

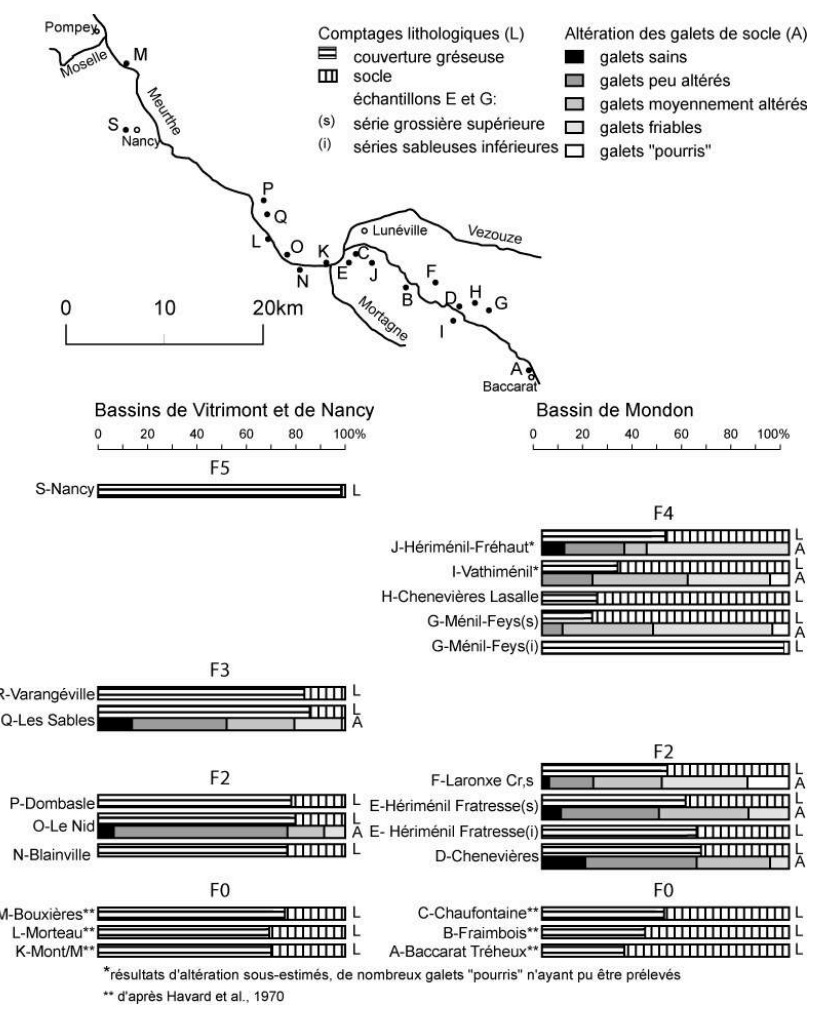

Compte tenu de ces caractéristiques communes (contraste entre un remblaiement sableux épais dans lequel dominent les apports issus du Buntsandstein, et un dépôt sommital plus grossier où la part des éléments issus du socle est plus importante), de leur appartenance à des terrasses situés à + 30-35 m d'altitude relative au-dessus du talweg, les cinq coupes décrites autorisent à définir un niveau alluvial-repère dans le bassin de Mondon.

Des observations topographiques et le dépouillement de sondages permettent par ailleurs de retrouver ce niveau en aval, avec toutefois une inversion du granoclassement: à l'aval de Lunéville, si la formation F4 reste essentiellement sableuse, les alluvions les plus grossières tendent à se localiser à la base du dépôt. Ce fait, qui ressort nettement dans l'étude des sondages réalisés dans l'agglomération de Nancy, traduit l'influence des affluents de la Meurthe (cf. infra).

De par l'épaisseur de ses alluvions et son extension spatiale, le niveau Me4 constitue néanmoins un niveau-référence pour l'identification du dispositif alluvial dans l'ensemble du secteur étudié. 


\section{Comparaison entre la formation F4 et les autres formations}

\section{A. Prédominance du faciès sableux et inversion du granoclassement}

Les faciès des autres formations (exemple de la coupe de Laronxe-Crâs, rattachée à F2, fig. 7) apparaissent au même titre que ceux de F4 comme nettement sableux, et ce dans toute la vallée.

Figure 7 : Synthèse sédimentologique des coupes de Laronxe-Crâs et de Ménil-Feys : logs (a), granulométrie (b), comptages lithologiques minéralogiques (c)

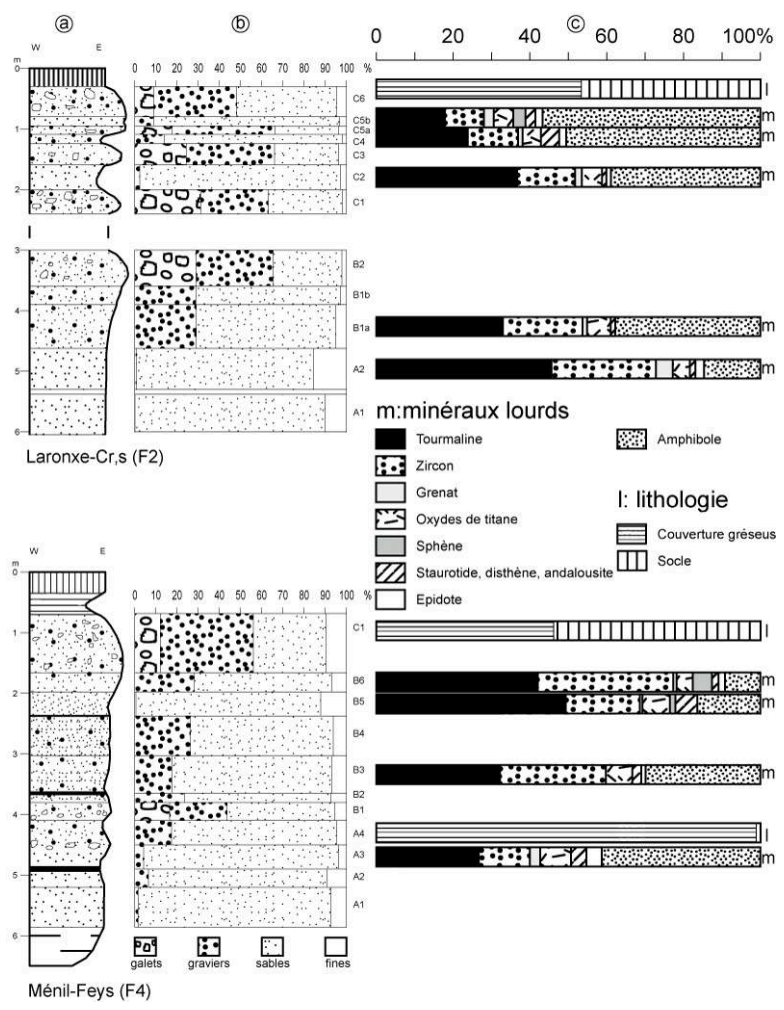

Dans les terrasses du bassin de Mondon, les niveaux grossiers apparaissent au sommet du dépôt. A l'aval, l'inversion granulométrique déjà évoquée pour $\mathrm{F} 4$ se retrouve : dans le bassin de Vitrimont, et surtout dans celui de Nancy (où l'étude s'appuie notamment sur l'analyse d'un grand nombre de sondages), le granoclassement est positif, conformément à ce qui a été observé pour le remblaiement de fond de vallée F0 (Carcaud, 1992). La seule nuance est la taille des éléments, la formation F0 étant souvent plus grossière que les alluvions anciennes.

Cette inversion granulométrique peut être corrélée avec les apports grossiers des deux principaux affluents rejoignant la Meurthe près de Lunéville, la Vezouze et surtout la Mortagne. Cette dernière a en effet (comme l'attestent des observations de terrain) une compétence élevée, liée à une pente forte. 


\section{B. Une double évolution de la nature des alluvions}

31 L'origine vosgienne des sédiments et la prédominance des apports grésoconglomératiques dans toutes les formations sont confirmées par l'ensemble des analyses minéralogiques (prépondérance de l'association tourmaline-zircon et secondairement de l'amphibole, fig. 5) et pétrographique (teneurs élevées en galets de quartz et quartzite, fig. 6).

Des évolutions apparaissent cependant, en fonction d'abord de l'ancienneté des alluvions, ensuite depuis l'amont vers l'aval :

1. Selon l'âge de la formation: l'étude minéralogique montre que la plaine alluviale se caractérise par un cortège diversifié avec l'amphibole, le grenat, le pyroxène et les oxydes de titane; la formation F5 ancienne a en revanche un cortège peu varié (prépondérance des ubiquistes) et l'amphibole, altérable, est rare. Les formations F2, F3 et F4 ont des cortèges comparables, mais le rapport tourmaline/zircon permet de les distinguer : ce rapport est inférieur à 1,5 pour F2 et F3 et avoisine 1,7 pour F4. (L'explication de cette variation pourrait être à rechercher dans une variation de la source gréseuse de ces sédiments : Sindowski (in Perriaux, 1961) note en effet que tourmaline et zircon - minéraux résistants et stables dominent tour à tour selon que les courants de sédimentation proviendraient principalement du Buntsandstein moyen, riche en tourmaline, ou des parties inférieures et supérieures de cet étage, riches en zircon). Les comptages lithologiques (fig. 6) font de même apparaître une évolution nette selon l'âge relatif des niveaux : on constate globalement une diminution de la proportion de galets de socle vers les formations anciennes (hormis dans le bassin de Mondon où ces galets sont encore abondants dans la formation F4). Cette tendance est liée à l'altération des granites au cours du temps, dont témoigne le contraste entre la formation F2 qui présente de nombreux galets peu altérés, et les formation F3 et surtout F4, où les galets sont fortement altérés.

2. D'amont en aval : pour une même formation alluviale, les bassins de Vitrimont et de Nancy se distinguent du bassin de Mondon par une proportion plus faible en éléments issus du socle cristallin: le cortège minéralogique montre ainsi une baisse de la proportion d'amphibole (notamment dans le bassin de Nancy). Surtout, le cortège pétrographique s'appauvrit considérablement en galets de socle: ceux-ci, souvent majoritaires dans le bassin de Mondon, représentent à l'aval moins de $40 \%$ voire $30 \%$ des galets. Cette évolution peut s'expliquer par l'éloignement croissant des affleurements cristallins, ainsi que par l'usure plus rapide des galets de granite lors de leur transport. À l'instar de l'inversion du granoclassement, elle paraît cependant surtout liée aux apports gréseux de la Mortagne, qui transporte de nombreux galets de quartz et quartzite.

Ainsi, la comparaison entre les formations alluviales de la Meurthe confirme le caractère nettement sableux du remblaiement évoqué pour F4, et permet de mettre en évidence une double évolution: d'une part, la nature et l'altération des alluvions varient avec l'ancienneté de la formation; par ailleurs, les caractéristiques de l'alluvionnement sont modifiées à l'aval de Lunéville, où l'on observe une inversion du granoclassement et une forte baisse de la teneur en éléments cristallins. 


\section{Alluvionnement de la Meurthe et glaciations vosgiennes : essai de reconstitution paléoclimatique}

\section{A. Dans le bassin de Mondon}

34 Une étude plus fine des résultats minéralogiques et pétrographiques permet de préciser le contraste précédemment décrit dans le bassin de Mondon entre remblaiement fin et grossier. Les exemples de Ménil-Feys (F4) et de Laronxe-Crâs (F2) sont particulièrement révélateurs (fig. 7)

35 A Ménil-Feys, où les comptages ont été effectués sur des échantillons issus des séquences sableuses, on constate de la base vers le sommet des séries fines une augmentation du pourcentage de tourmaline et de zircon (de 40 à $77,3 \%$ ), alors que celui de l'amphibole baisse de 41,3 à 9,2\%. L'augmentation de la part de minéraux issus de la couverture permo-triasique indique que l'alluvionnement a une origine de plus en plus proximale par rapport à l'ensemble du bassin de sédimentation.

36 A l'opposé, à Laronxe-Crâs, les échantillons ont été prélevés dans le remplissage grossier, à l'exception de A2. Or, les comptages montrent nettement une diminution vers le haut de la proportion en tourmaline et du zircon. Au contraire, la proportion d'amphibole passe de 14,7 à $56,7 \%$ : la mise en place du remplissage grossier correspond donc à des apports de plus en plus « distaux » (Vosges cristallines).

37 Deux lithofaciès doivent donc être distingués dans les formations des terrasses de la Meurthe dans le bassin de Mondon:

1. un lithofaciès "proximal», sableux et riche en tourmaline et en zircon (ce faciès, très développé à Ménil-Feys, se retrouve à la base de la coupe de Laronxe-Crâs). Les galets (quartz et quartzites) que l'on y trouve sont également issus du Buntsandstein ;

2. un lithofaciès «distal», plus grossier et riche en éléments cristallins, qui apparaît très nettement à Laronxe-Crâs. Bien que ce faciès soit moins épais à Ménil-Feys, la forte proportion de galets cristallins observée dans la série $C$ est révélatrice de l'origine distale des sédiments (il n'a pas été possible, faute d'échantillonnage, de mettre en évidence la présence de minéraux issus du socle dans les sables de cette série C).

38 Cette dualité peut être corrélée avec les variations climatiques ayant affecté le Massif vosgien au Quaternaire (fig. 1), le bassin supérieur de la Meurthe ayant été englacé à plusieurs reprises (Darmois-Théobald et Menillet, 1973 ; Menillet, 1975). Le dépôt du lithofaciès " proximal » est relié à des périodes où la Meurthe a une capacité de transport modérée, son bassin amont étant englacé. De plus, l'observation de figures de cryoturbation (plications et involutions) dans ces niveaux (formation F3, Cordier et al., soumis), laisse supposer une mise en place en phase pléniglaciaire. Le faciès "distal» correspond pour sa part à une sédimentation en haut régime d'écoulement, avec un débit liquide élevé et une compétence forte typiques de périodes de déglaciations. Compte tenu des caractéristiques du bassin et des observations récentes dans la plaine alluviale de la Meurthe (Carcaud, 1992), dans la vallée de la Moselle (Taous, 1994) ou de la Meuse (Vandenberghe et al., 1994), ce lithofaciès « distal » peut être rattaché aux phases tardiglaciaires, ce qui rejoint les conclusions de Carcaud (1992) pour la formation F0. La formation limono-sableuse recouvrant localement ce lithofaciès correspondrait ainsi aux restes des dépôts interglaciaires. 
39 Dès lors, l'incision majeure survient vraisemblablement lors de la dégradation climatique: elle peut en effet difficilement être attribuée aux périodes tardiglaciaires, comme en témoigne l'absence d'incision majeure à Holocène dans la formation F0 d'âge Tardiglaciaire (Carcaud et al., 1991).

\section{B. Dans les bassins de Vitrimont et Nancy}

40 A l'aval de Lunéville, l'inversion granulométrique constatée paraît liée à l'influence de la Mortagne. Cet affluent de la Meurthe, dont le bassin n'a pas été occupé par des glaciers (fig. 1), transporte en effet de nombreux éléments grossiers. La mise en place de ces dépôts peut être attribuée aux périodes froides, à l'image des résultats obtenus lors de l'étude de nombreux cours d'eau périglaciaires (Sommé, 1984; Antoine, 1994).

41 Lors des épisodes pléniglaciaires, les apports sableux de la Meurthe (séries A et B du bassin de Mondon) se mêlent ainsi aux galets de la Mortagne, d'où une sédimentation grossière à la base. Celle-ci se poursuit lors des épisodes tardiglaciaires (série $\mathrm{C}$ ), avant que le retour à des conditions plus tempérées ne se traduise par des dépôts plus fins, d'où un granoclassement d'ensemble positif.

\section{C. Âge présumé des dépôts}

42 A titre d'hypothèse ( $\mathrm{du}$ fait de l'absence d'éléments de datation décisifs), et par analogie avec les résultats obtenus dans la vallée de la Moselle touloise (Harmand et al., 1995, Cordier et al., 2002), la chronologie suivante de mise en place des formations alluviales F1 à F4 peut être proposée: F4 serait déposée au Saalien moyen (stade isotopique 8), F3 au Saalien supérieur (stade 6), F2 au Pléniglaciaire ancien (stade 4) et F1 au Weichsélien moyen (stade 2).

\section{Conclusions}

43 La présente étude permet de mieux appréhender les principales caractéristiques de l'alluvionnement dans la vallée de la Meurthe à l'aval de Baccarat :

1. le dispositif alluvial se compose de 10 terrasses réparties entre la plaine alluviale et $160 \mathrm{~m}$ d'altitude relative ;

2. l'alluvionnement est essentiellement sableux, à l'exception d'un remplissage grossier dans lequel se trouvent des éléments cristallins issus du bassin supérieur de la Meurthe, englacé à plusieurs reprises au Quaternaire ;

3. alors que les dépôts sableux témoignent pour le bassin de Mondon d'une sédimentation pléniglaciaire (faciès " proximal »), la mise en place de la série grossière (faciès « distal ») est attribuée aux épisodes tardiglaciaires ;

4. à l'aval, le remplissage grossier observé à la base des alluvions semble correspondre d'abord à une sédimentation lors des périodes froides ("pléniglaciaires»), liée principalement à laMortagne, puis à une sédimentation lors des épisodes tardiglaciaires (apports de la Meurthe). La compétence de la rivière diminue ensuite avec le retour à des conditions climatiques plus tempérées, d'où le granoclassement positif observé ; 
5. la composition des sédiments varie également d'amont en aval, les éléments cristallins étant moins abondants à l'aval de Lunéville, du fait principalement des apports grésoconglomératiques grossiers de la Mortagne.

\section{BIBLIOGRAPHIE}

Allen J.R.L. (1982). - Sedimentary structures: their character and physical basis. Developments in sedimentology, 30, Elsevier, $663 \mathrm{p}$.

Antoine P. (1994). - The Somme valley terrace system (Northern France) : a model of river response to Quaternary climatic variations since 800000 B.P. Terra Research, 5, pp. 453-464.

Bonnefont J.C. (1986). - Les cailloutis anciens du piémont occidental des Vosges et leur signification géomorphologique. Revue géogr. de l'Est, $\mathrm{n}^{\circ} 2$, pp. 71-80.

Buzzi P., Carcaud N., Koenig M.P., Weisrock A. (1993). - Morphodynamique fluviale holocène et établissements humains protohistoriques en fond de vallée de la Moselle à Crévéchamps (Lorraine méridionale), in « Vallée fluviales en Europe à l'Holocène ». Revue géographique de l'Est, t. 33, n 4, pp. 281-295.

Caillier M. (1977). - Etude chronoséquentielle des sols sur terrasses alluviales de la Moselle. Genèse et évolution des sols lessivés glossiques. Thèse spécialité Pédol., Univ. Nancy I, 87 p.

Carcaud N. (1992). - Remplissage des fonds de vallée de la Moselle et de la Meurthe en Lorraine sédimentaire. Thèse, Université de Nancy 2, $281 \mathrm{p}$.

Carcaud N., Weisrock A., Occhietti S. (1991). - Creusements et alluvionnements holocènes de la Meurthe et de la Moselle. Physio-Géo, n 22-23, pp. 131-136.

Cordier S., Harmand D., Losson B., Beiner M. (2002). - Pleistocene accretion in the Eastern Paris Basin : lithofacies and incision rhytms in the Meurthe and Moselle valleys. Livret des résumés, Fluvial Archives Group (FLAG) biennal meeting, Clermont-Ferrand, 9-11 septembre 2002, p. 12.

Cordier S., Harmand D., Losson B., Beiner M. - Pleistocene alluviation in the Meurthe and the Moselle valleys (eastern Paris basin) : lithofacies and incision rhytms, first results. Quaternaire, article soumis.

Darmois-Theobald M., Menillet F. (1973). - Recherches sur la morphologie glaciaire des vallées supérieures de la Meurthe (Vosges). Ann. Sci. Univ. Besançon, fasc. 21, pp. 113-128.

Doeglas D. J. (1962). - The structure of sedimentary deposits of braided rivers. Sedimentology, 1, pp. 167-190.

Gury M. (1990). - Genèse et fonctionnement actuel des pseudogleys podzoliques sur terrasses alluviales dans l'Est de la France. Thèse Univ. Nancy I, 331p.

Hameurt J. (1967). - Les terrains cristallins et cristallophylliens du versant occidental des Vosges moyennes. Mém. Serv. Carte Géol. Als.-Lorr., 26, Strasbourg, 402 p.

Harmand D., Le Roux J. (2000). - La capture de la Haute Moselle. Bull. Inf. Géol. Bassin Paris., vol. 37, n³, pp. 4-14. 
Harmand D., Pissart A., Krook L. (1998). — L'évolution du paléo-bassin de la Meuse : les enseignements des captures et leurs implications environnementales. Arbeiten aus dem Geographischen Institut der Universität des Saarlandes, Symposium « problèmes de l'environnement en Saar-Lor-Lux », pp. 157-173.

Harmand D., Weisrock A., Gamez P., Le Roux J., Occhietti S., Deshaies M., Bonnefont J.C., Sary M. (1995). - Nouvelles données relatives à la capture de la Moselle. Revue Géographique de l'Est, Nancy, t. XXXV, 3-4, pp. 321-343.

Havard H., Mauget G., Husson B. (1970). - Etude des matériaux alluvionnaires du bassin versant de la Moselle. Laboratoire des Ponts et Chaussées de Nancy, 173 p.

KowalskI P. (1988). - Contribution à l'étude des nappes alluviales de la Meurthe. La nappe alluviale du Bozey à Sainte-Marguerite (Vosges). Rapport de DEA Univ. Nancy 2, 46 p.

Kowalski P., Taous A., Weisrock A. (1990). - Etude morphosédimentaire du remblaiement de fond de vallée de la Meurthe à Sainte-Marguerite (Vosges) : comparaison avec la Moselle à Chavelot (Vosges) et premiers enseignements. Revue géogr. de l'Est, t. 30, n 1, pp. 19-36.

Kremer E. (1954). - Die Terrassenlandschaft der mittleren Mosel als Beitrag zur Quartärgeschichte., Arbeiten zur Rheinischen Landeskunde, Heft 6, 91 p.

Lebret P., Campy M., Coutard J.P., Fourniguet J., Isambert M., Lautridou J.P., Laville P., Macaire J.J., Menillet F., Meyer R. (1993). - Cartographie des formations superficielles : réactualisation des principes de représentation à 1/50 000. Géologie de la France, 4, pp. 39-54.

Le Roux J. (1980). - « La tectonique de l'auréole orientale du Bassin de Paris. Ses relations avec la sédimentation », Bull. Soc. Géol. France, 7, t. XXII, n 4, pp. 655-662.

Le Roux J., Harmand D. (1998). - Contrôle morphostructural de l'histoire d'un réseau hydrographique : le site de capture de la Moselle. Geodinamica acta, vol. 11, nº 4, pp. 149-162.

Menillet F. (1975). - Notice explicative de la carte géologique de France au 1/50 000e , feuille de Saint-Dié, formations superficielles. Publ. du BRGM.

Mercier J.L., Bourles D., Kalvoda I., Vergne V., Braucher R., Pachen A ., Raisbeck G.M., Yiou F. (1999). - 10Be dating of moraines and roches moutonnées at low altitude in continental Europe during the Holocène. Geophysical Research Abstracts, vol. 1, n 2, p. 576.

Miall A.D. (1996). - The geology of fluvial deposits: sedimentary facies, basin analysis and petroleum geology. Berlin, New-York, Springer, 1996, 582 p.

Perriaux F. (1961). - Contribution à la géologie des Vosges gréseuses. Mém. Serv. Carte Géol. AlsLorr., 18, Strasbourg, $236 \mathrm{p}$.

Schirmer W. (1990). - Rheingeschichte zwischen Mosel und Maas. Deutsche Quartärvereinigung, Hannover, $259 \mathrm{p}$.

Somme J. (1984). - Signification dynamique et climatique des formations et terrasses fluviatiles dans l'Europe du Nord-Ouest. Bulletin de l'AFEQ, 1-2-3, pp. 9-12.

Taous A. (1994). - Le système alluvial de la moyenne terrasse de la Moselle en Lorraine méridionale (approche sédimentaire et pétrographique). Univ. Nancy 2, thèse, non publiée, 201 p. Theobald N., Gardet G. (1935). - Les alluvions anciennes de la Moselle et de la Meurthe en amont de Sierck. Bull. Cent. Soc. Hist. Nat. de Metz, 34e Bull., sér. 3, t. 10, pp. 69-100.

Tricart J. (1948). - La partie orientale du Bassin de Paris, étude morphologique. Thèse doct., SEDES, Paris, 2 t., 210 et $64 \mathrm{p}$. 
Vandenberghe J., Kasse C., Bohncke S., Kozarski S. (1994). - Climate-related river activity at the Weichselian-Holocene transition: a comparative study of the Warta and Maas rivers. Terra Nova, 6, pp. 476-485.

Vaskou P. (1981). - Apports de la sédimentologie à la connaissance des dépôts alluviaux des terrasses de la Moselle entre Noirgueux et Toul. Thèse, Univ. Nancy I, 145 p.

Vogt H. (1992). - Le relief en Alsace : Etude géomorphologique du rebord sud-occidental du fossé rhénan. Oberlin, Strasbourg, $239 \mathrm{p}$.

Weisrock A. (1990). - Introduction à l'étude de la genèse des fonds de vallées en Lorraine. Revue géogaphique de l'Est, t. 30, n 1, pp. 3-18.

Weisrock A. (1997). - Cent ans après Davis : la question des captures et les paléoréseaux hydrographiques quaternaires d'après les exemples de l'Europe du Nord-Ouest. Géographie physique et Quaternaire, 51, 3, pp. 261-266.

\section{RÉSUMÉS}

À l'aval du Massif vosgien, la Meurthe présente un ensemble de terrasses bien conservé, constitué de 10 niveaux de terrasses étagés. L'analyse de coupes, notamment pour le niveau Me4 (30 m d'altitude relative), permet de caractériser l'alluvionnement de la Meurthe. Celui-ci est essentiellement composé d'un remblaiement sableux issu de la couverture permo-triasique des Vosges. Les sédiments plus grossiers, peu épais et riches en éléments issus du socle hercynien, sont observés en sommet de remplissage à l'amont de Lunéville, tandis qu'ils constituent la base du remblaiement plus en aval. La mise en place des dépôts sableux est attribuée aux épisodes pléniglaciaires, celle des séries grossières aux épisodes tardiglaciaires.

Downstream from the Vosges Massif, the Meurthe river valley contains a sequence of ten wellpreserved terrace levels. The study of several exposures, notably in terrace Me4 (30 m of relative altitude) facilitates characterisation of Meurthe alluviation, this one essentially composed of sandy material derived from the Permo-Triassic cover of the Vosges. Coarser sediments, of limited thickness and rich in material derived from the Hercynian basement, occur at the top of the sequence upstream of Lunéville while they constitute the base of the sequence further downstream. Deposition of the sandy deposits and the coarser deposits probably occurred during full-glacial and late-glacial episodes, respectively.

Unterhalb der Vogesen weist das Tal der Meurthe eine Terrassentreppe auf, die sich in 10 Niveaus aufteilen läßt. Untersuchungen an Gruben in Terrassenkörpern, insbesondere in der $30 \mathrm{~m}$ Terrasse (Me4) erlauben, die Ablagerung der Schotter im Meurthetal zu kennzeichnen. Oberhalb von Lunéville bestehen die Ablagerungen aus einer mächtigen Schicht von Sanden, die aus der Buntsandsteinumrahmung der Vogesen kommen und auf diesen Sanden liegen Schotter, die aus dem Grundgebirge stammen. Es wird daraus geschlossen, dass die sandigen Ablagerungen und die Schotter während bzw. nach der Hochkaltzeit abgelagert wurden. 
INDEX

Keywords : alluvial terraces, fluvial deposits, glacial cycle, heavy minerals, Lorraine, Meurthe Mots-clés : cycle glaciaire, depôts fluviatiles, Lorraine, Meurthe, minéraux lourds, terrasses alluviales

Schlüsselwörter : AlluvialTerrassen, Aufschüttungen, glazialzyklus, Lothringen, Meurthe, Schwermineralanalysen

\section{AUTEURS}

\section{STÉPHANE CORDIER}

Géodynamique des milieux naturels et de l'environnement, Faculté des Lettres et Sciences Humaines, Université de Paris XII, 61, avenue du Général de Gaulle, 94010 Créteil Cedex ; scordier@club-internet.fr

\section{DOMINIQUE HARMAND}

Laboratoire de Géographie, Faculté des Lettres et Sciences Humaines, Université de Nancy 2, BP 33-97, Nancy ; Dominique.Harmand@univ-nancy2.fr

\section{MONIQUE BEINER}

2a, rue du jardin Keck, 67000 Strasbourg ; Beinerjm@aol.com 